\title{
Design and Optimization of a Low Cost MEMS Piezorezistive Pressure Transducer for Pneumatic Systems
}

\author{
A. Popescu-Cuta, O.G. Dontu, C.D. Comeaga and V.I. Gheorghe
}

\begin{abstract}
MEMS sensors registered a constant increase in pneumatic intelligent systems, due to their excellent performance, low costs and small size. The most widely used MEMS pressure transducers are the ones based on the piezoresistive effect, due to their high linearity and excellent characteristics. As the performance of these transducers is highly influenced by their constructive parameters, a design optimization of a piezoresistive MEMS pressure transducer has been performed using the COMSOL Multiphysics 5.0 software.
\end{abstract}

Index Terms - COMSOL Multiphysics simulation, finite element analysis, MEMS piezoresistive sensor.

\section{INTRODUCTION}

Pressure is one of the critical parameters that needs to be monitored in order to obtain a precise control of an intelligent pneumatic system. Pressure measurement is achieved by using dedicated equipment, called pressure transducers, which are able to transform the input pressure into an electrical signal. As the trend to miniaturization is continuously evolving, the classical pressure transducers are being replaced with MEMS devices, able to deliver the information with high precision, linearity and response time [1].

The most widespread type of MEMS pressure transducers are based on the piezoresistive effect, which involves a shift in the electrical resistance of the sensing material, as consequence of strain. The deformation of the sensing material is caused by the application of an input pressure, therefore the electrical output of the piezoresistive pressure sensors will be proportional with the applied pressure [2].

The performance of the MEMS piezoresistive pressure transducers depends on several factors, as temperature compensation, materials and constructive parameters. The shape of the sensing membrane, its thickness and also the position of the piezoresistors have been proven to have a significant impact over the transducer performance and sensitivity in a certain pressure interval [3].

The paper presents the optimization of a MEMS piezoresistive pressure transducer, designed for pneumatic

Manuscript received April 1st, 2016. This work was partially supported by the strategic grant POSDRU/159/1.5/S/137070 (2014) of the Ministry of National Education, Romania, co-financed by the European Social Fund Investing in People, within the Sectorial Operational Program Human Resources Development 2007-2013.

A. Popescu-Cuta is with the Politehnica University of Bucharest, Splaiul Independenței, no. 313, District 6, Bucharest, Romania

O. G. Dontu is with the Politehnica University of Bucharest, Splaiul Independenței, no. 313, District 6, Bucharest, Romania

C. D. Comeaga is with the Politehnica University of Bucharest, Splaiul Independenței, no. 313, District 6, Bucharest, Romania

V. I. Gheorghe is with the Politehnica University of Bucharest, Splaiul Independenței, no. 313, District 6, Bucharest, Romania systems. As in pneumatics the pressure range varies from 1 to 10 bar, the proposed transducer has been designed for an optimal sensitivity in this interval. In order to determine the optimal geometric configuration of the transducer, various simulations of the sensor behavior have been performed using the COMSOL Multiphysics 5.0 software.

\section{THE STUDIED TRANSDUCER}

Different configurations of MEMS pressure transducers have been previously studied in several papers, as [4]. In the model presented in [4], two of the piezoresistors have been positioned transversally and two have been placed longitudinally. The model studied in this paper presents a different configuration, with all the piezoresistors positioned transversally, in order to determine if a proper linearity can be achieved also by using a different geometry.

The model used in paper [4] implies the need of two masks for the piezoresistor deposition, as the dimensions and positions of the sensing elements are different. By using the configuration presented in the following paper, only one mask is necessary and all the resistors can be placed in the same time, consequently simplifying the technological process and leading to lower fabrication costs.
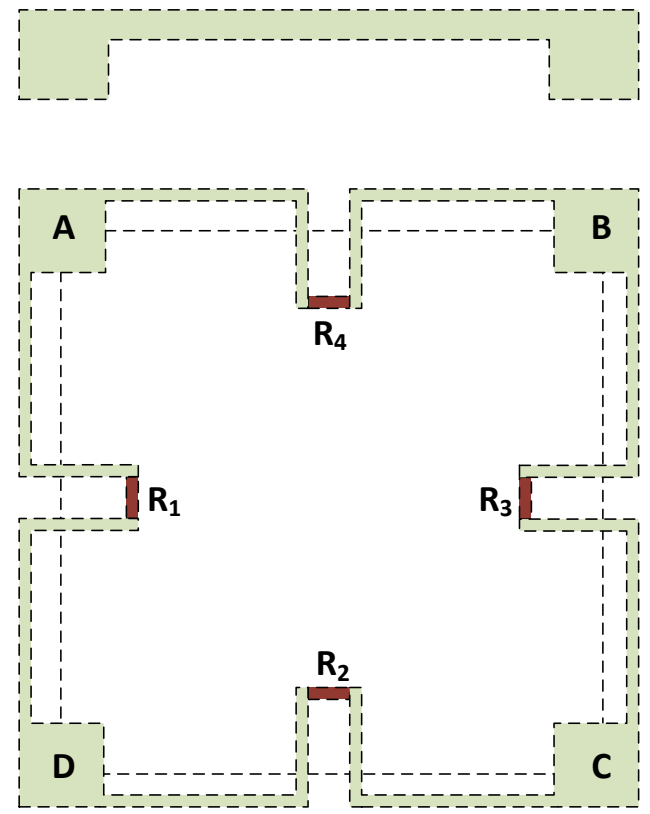

Fig. 1. The sensor configuration.

The piezoresistors $\left(\mathrm{R}_{1}, \mathrm{R}_{2}, \mathrm{R}_{3}, \mathrm{R}_{4}\right)$ are marked with red color and the terminals for applying voltage and reading the output are named A, B, C, D. The sensor chip is square shaped, measuring $3000 \mu \mathrm{m} \times 3000 \mu \mathrm{m}$ and the thickness of the chip is 
$100 \mu \mathrm{m}$. The sensor membrane is also square, measuring 2000 $\mu \mathrm{m}$ x $2000 \mu \mathrm{m}$. The proper membrane thickness for measurements of the pressures varying in the $1 . .10$ bar interval, will be determined throughout the study.

According to [4], the deformation waves become almost linear near the fixed borders of the sensor, so consequently the piezoresistors should be placed closer to the margins of the membrane and not close to the center of the sensor. The proper position of the piezoresistors will also be determined throughout the study.

Under the effect of strain, a shift in the electrical resistance of the four piezoresistors occurs. Equation (1) describes the relation between silicon resistivity change and stress [5]:

$$
\frac{1}{\rho}\left[\begin{array}{c}
\Delta \rho_{1} \\
\Delta \rho_{2} \\
\Delta \rho_{3} \\
\Delta \rho_{4} \\
\Delta \rho_{5} \\
\Delta \rho_{6}
\end{array}\right]=\left[\begin{array}{cccccc}
\pi_{11} & \pi_{12} & \pi_{12} & 0 & 0 & 0 \\
\pi_{12} & \pi_{11} & \pi_{12} & 0 & 0 & 0 \\
\pi_{12} & \pi_{12} & \pi_{11} & 0 & 0 & 0 \\
0 & 0 & 0 & \pi_{44} & 0 & 0 \\
0 & 0 & 0 & 0 & \pi_{44} & 0 \\
0 & 0 & 0 & 0 & 0 & \pi_{44}
\end{array}\right]\left[\begin{array}{l}
\sigma_{1} \\
\sigma_{2} \\
\sigma_{3} \\
\tau_{1} \\
\tau_{2} \\
\tau_{3}
\end{array}\right],
$$

where:

$\rho=$ resistivity;

$\pi_{i, j}=$ piezoresistive coefficients;

$\sigma_{i}=$ stress;

$\tau_{i}=$ shear stress

Consequently, the change in the piezoresistors resistance is described by (2), as following:

$$
\frac{\Delta R}{R}=\frac{\Delta \rho}{\rho}=\pi_{1} \sigma_{l}+\pi_{t} \sigma_{t}
$$

where:

$R=$ electrical resistance

Considering the fact that all the piezoresistors are subjected to in-plane stress, it can be concluded that all of them register an increase of the resistance with strain. But as all the resistors have been deposed with the same orientation and silicon is an anisotropic material, the resistance variation is different for each pair of resistors, due to their different sensitivities at the same strain (the sensitivity of the $R_{1} / R_{3}$ pair of resistors is different than the sensitivity of $R_{2} / R_{4}$ pair), determined by the anisotropy of silicon.

The resistors are connected in a Wheatstone bridge (Fig. 2), which is balanced when the resistors are not subjected to strain. As pressure is applied, the different increase in resistance for each pair of sensitive elements determine the unbalance of the Wheatstone bridge and therefore, an output voltage, proportional with the resistance change and the applied pressure.

The $R_{1}$ and $R_{3}$ elements exhibit a greater variation of resistance with strain, than $R_{2}$ and $R_{4}$.

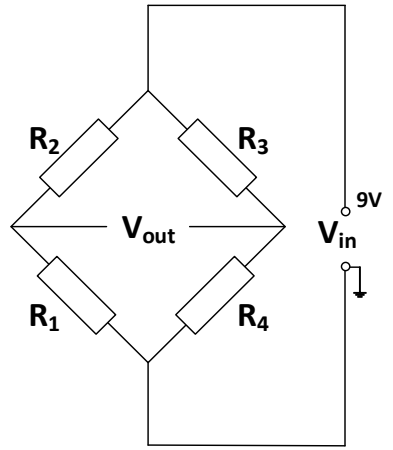

Fig. 2. The four piezoresistors connected in a Wheatstone bridge.

\section{MODELING USING COMSOL MULTIPHYSICS}

A model of the transducer has been created using the COMSOL Multiphysics software, Piezoresistivity and Boundary currents module.

The first step was to create a block with the given dimensions for width, length and height. Following, the sensor membrane was created, by extruding a hole in the created block. The extrude depth has been defined parametrically, so that the thickness of the membrane could be varied during the study.

The piezoresistors and the connections have been designed using a 2D drawing on the face of the block. The position of the piezoresistors has also been parametrically defined, in order to determine its best value for the given pressure interval. The thickness of both the piezoresistive and the conductive layers has been set to $400 \mathrm{~nm}$.

The material of the membrane and frame is defined as lightly doped n-silicon and the piezoresistors are built from lightly doped p-silicon. The material used for creating the electrical connections is Aluminium, from the MEMS materials database.

The complete model of the transducer is presented in Fig.3.

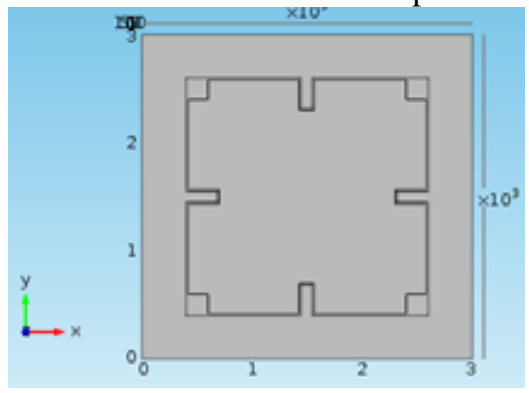

(a)

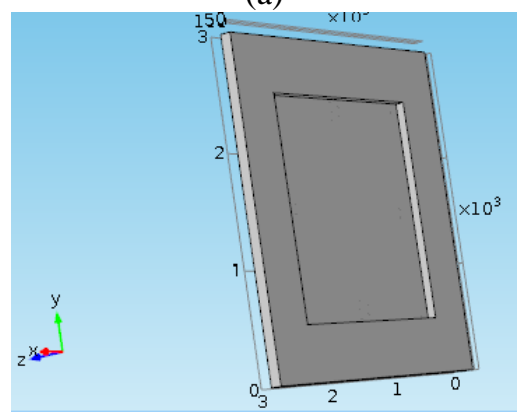

(b)

Fig. 3. The sensor model, created in COMSOL Multiphysics, (a)-front, (b)- back. 
In order to perform the study, the rim of the sensor has been defined as fixed. After that, a boundary load defined as pressure has been applied on the sensor membrane, on the face opposed to the piezoresistors. The connections between the piezoresistors have been defined as thin conductive layers and the resistors as piezoresistive layers.

A 9V voltage has been added to terminal C, while terminal A has been defined as ground. The output of the sensor will be read between terminals $\mathrm{B}$ and $\mathrm{D}$.

As the geometry of the structure is relatively simple, a physics controlled fine mesh has been built. The meshing type ensures a short calculation time, reducing the need for advanced hardware in order to perform the study. The meshed model of the sensor is presented in Fig. 4.

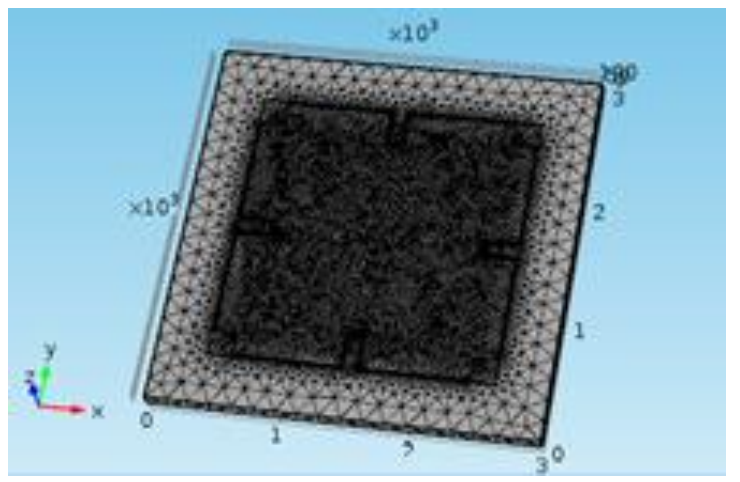

Fig. 4 The meshed sensor model.

\section{RESULTS AND DISCUSSIONS}

The first step of the study was to determine the proper thickness of the sensing membrane, for pressures ranging from 1 to 10 bar. Therefore, a parametric study has been completed, by varying the extrusion depth in the sensor plate, which determines the thickness of the membrane. The depth has been varied between 10 and $80 \mu \mathrm{m}$, corresponding to membrane thicknesses of 90 to $20 \mu \mathrm{m}$, at an input pressure of $1 \mathrm{bar}$. The study had the purpose to determine the optimal thickness of the membrane, at which the highest voltage output is obtained at the minimum input pressure.

The results of the study are presented in Table 1 and the graphic variation of the output voltage with the membrane thickness is presented in Fig. 5.

TABLE I

The Variation Of THE OUtPut Voltage With THE MEMBRANE THICKNESS

\begin{tabular}{ll}
\hline \hline Membrane thickness $[\mu \mathrm{m}]$ & Output voltage $[\mathrm{V}]$ \\
\hline 20 & $9,99 \mathrm{E}-05$ \\
30 & $1,54 \mathrm{E}-05$ \\
40 & $7,11 \mathrm{E}-05$ \\
50 & $1,36 \mathrm{E}-05$ \\
60 & $-7,36 \mathrm{E}-06$ \\
70 & $9,65 \mathrm{E}-07$ \\
80 & $-1,08 \mathrm{E}-06$ \\
90 & $6,75 \mathrm{E}-07$ \\
\hline \hline
\end{tabular}

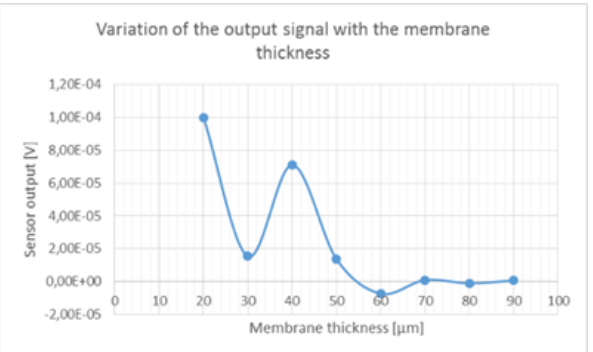

Fig. 5 The variation of the output voltage with the membrane thickness.

As seen in Fig. 5, the highest output for the minimal targeted pressure (1 bar) is registered at an extrusion depth of $80 \mu \mathrm{m}$, which corresponds to a membrane thickness of $20 \mu \mathrm{m}$. Therefore, the optimal membrane thickness of the proposed sensor will be considered $20 \mu \mathrm{m}$.

The next study had as purpose the determination of the optimal position of the piezoresistors for which the highest output is obtained for the minimal pressure. A parametric sweep of the resistors position in report with the membrane edge has been computed, for values ranging from $100 \mu \mathrm{m}$ to $500 \mu \mathrm{m}$ and a step of $50 \mu \mathrm{m}$. The input pressure was maintained constant at 1 bar and the membrane thickness was considered $20 \mu \mathrm{m}$. The results of the study are presented in Table 2 and Fig. 6.

TABLE II

The Variation Of The Output Voltage With The Distance Between THE PIEZORESISTORS AND THE MEMBRANe EDGE

\begin{tabular}{ll}
\hline \hline $\begin{array}{l}\text { Distance from the } \\
\text { membrane edge }[\mu \mathrm{m}]\end{array}$ & Output voltage $[\mathrm{V}]$ \\
\hline 100 & $-6,75 \mathrm{E}-06$ \\
150 & $6,38 \mathrm{E}-06$ \\
200 & $-1,07 \mathrm{E}-04$ \\
250 & $-4,57 \mathrm{E}-05$ \\
300 & $9,92 \mathrm{E}-05$ \\
350 & $1,38 \mathrm{E}-05$ \\
400 & $4,33 \mathrm{E}-05$ \\
450 & $1,22 \mathrm{E}-05$ \\
500 & $-2,00 \mathrm{E}-05$ \\
\hline
\end{tabular}

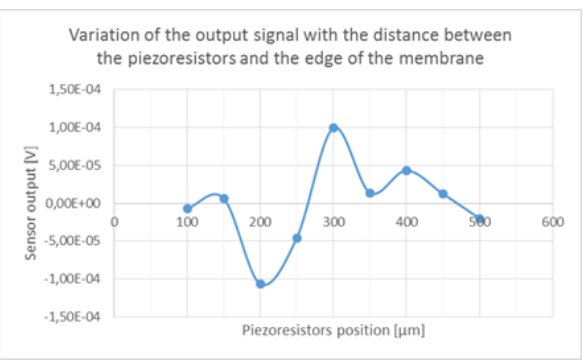

Fig. 6. The variation of the output voltage with the distance between the piezoresistors and the membrane edge

As seen in Fig. 6, the highest output at the minimum 1 bar pressure is registered when the piezoresistors are positioned at a $300 \mu \mathrm{m}$ distance from the edge of the membrane.

Based on the values obtained until now, a simulation of the piezoresistive pressure transducer is done, for the targeted pressure range of $1 \ldots 10$ bar. The membrane thickness was considered $20 \mu \mathrm{m}$ and the piezoresistors were placed at $300 \mu \mathrm{m}$ from the edge of the membrane. A parametric sweep of the input 
pressure has been computed, ranging from 1 to 10 bar, at a 0.5 bar step. The results of the study are presented in Table 3 and Fig. 7.

TABLE II

The Variation Of The Output Voltage With The Distance Between THE PIEZORESISTORS AND THE MEMBRANE EDGE

\begin{tabular}{ll}
\hline \hline Input pressure [bar] & Output voltage [V] \\
\hline 1 & $9,97 \mathrm{E}-05$ \\
1.5 & $1,06 \mathrm{E}-04$ \\
2 & $1,14 \mathrm{E}-04$ \\
2.5 & $1,24 \mathrm{E}-04$ \\
3 & $1,35 \mathrm{E}-04$ \\
3.5 & $1,46 \mathrm{E}-04$ \\
4 & $1,58 \mathrm{E}-04$ \\
4.5 & $1,73 \mathrm{E}-04$ \\
5 & $1,87 \mathrm{E}-04$ \\
5.5 & $2,03 \mathrm{E}-04$ \\
6 & $2,21 \mathrm{E}-04$ \\
6.5 & $2,38 \mathrm{E}-04$ \\
7 & $2,57 \mathrm{E}-04$ \\
7.5 & $2,78 \mathrm{E}-04$ \\
8 & $2,99 \mathrm{E}-04$ \\
8.5 & $3,22 \mathrm{E}-04$ \\
9 & $3,45 \mathrm{E}-04$ \\
9.5 & $3,70 \mathrm{E}-04$ \\
10 & $3,94 \mathrm{E}-04$ \\
\hline \hline
\end{tabular}

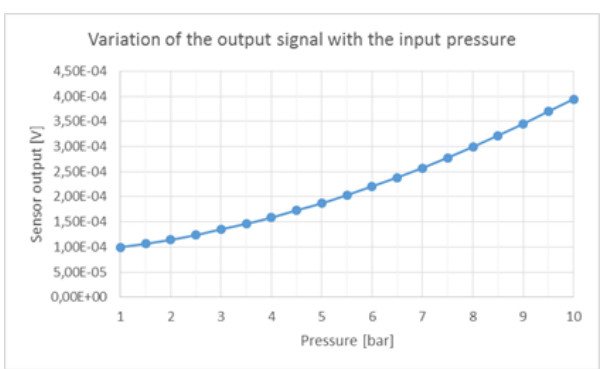

Fig. 7. Variation of the output voltage with the input pressure

The polynomial function describing the relation between the input pressure and the voltage output is presented in (3):

$$
y=2 \square 10^{-6} x^{2}+9 \square 10^{-6} x+9 \square 10^{-5}
$$

\section{CONCLUSION}

The article has highlighted the possibility of the constructive optimization of MEMS piezoresistive pressure sensors, regarding both the membrane parameters and the piezoresistors positions.

The study started from a simple geometrical structure, with a simple fabrication process using single crystal silicon etching.

The pressure - output electric signal characteristic is slightly nonlinear, but a simple mathematical relation can be established between the two parameters, which allows a software correction of the characteristic in the microcontroller associated to the transducer or in the external acquisition system.

\section{REFERENCES}

[1] A.A.S. Mohammed, W.A. Moussa and E. Lou, "High-Performance Piezoresistive MEMS Strain Sensor with Low Thermal Sensitivity" Sensors, vol. 11, pp. 1819-1846, 2011. http://dx.doi.org/10.3390/s110201819

[2] A. Barlian, W. T. Park, J. R. Mallon, A. J. Rastegar, and B. L. Pruitt, "Semiconductor Piezoresistance for Microsystems", Proceedings of the IEEE, Institute of Electrical and Electronics Engineers, pp. 513-552, vol. 97, 2009. http://dx.doi.org/10.1109/JPROC.2009.2013612

[3] N. A. Fathi and Z. A. Moradi,"'Design and Optimization of Piezoresistive MEMS Pressure Sensors Using ABAQUS", Middle-East Journal of Scientific Research, Volume 21, (2014).

[4] S. Meenatchisundaram, S. Kulkarni and S. Bhat: "Design Analysis and Sensitivity Enhancement of Piezoresistive Micro Pressure Sensors", Proceedings of 2015 International Conference on Future Computational Technologies (ICFCT'2015), pp. 104-110, Singapore, 2015.

[5] G. K. Johns, "Modeling piezoresistivity in silicon and polysilicon", Journal of Applied Engineering Mathematics, Volume 2, (2006).

[6] S. S. Kumar, A.K. Ojha, R. Nambisan, A.K. Sharma and B.D. Pant: "Design and Simulation of MEMS Silicon Piezoresistive Pressure Sensor for Barometric Applications", International conference on advances in recent technologies in Electrical and Electronics (ARTEE), India, 2013.

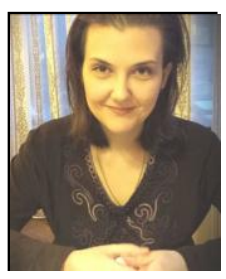

A. Popescu-Cuta was born in Pitesti, Romania, 1988. She received her BSc. in mechatronics from Politehnica University of Bucharest, Romania, in 2011 and her MSc in advanced mechatronics in 2013, from the same University.

Eng. Popescu-Cuta is currently a Ph.D. student in the Department of Mechatronics and Precision Mechanics, in the Politehnica University of Bucharest, Romania.

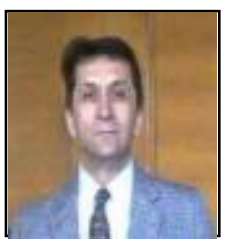

O.G. Dontu was born in Ploiesti Romania, 1947.

$\mathrm{He}$ received the BSc, in precision mechanics (fine mechanics) in 1970 from University Politehnica and obtained the PhD title in laser engineering systems for material processing in 1985 . He works as a professor at University Politehnica of Bucharest, Romania, Faculty of Mechanical Engineeringa and Mechatronics, the Department of Mechatronics. He is $\mathrm{PhD}$ supervisor and in the last 10 years taught classes on "Laser Processing Technology" at E.N.S.A.M. Aix en Provence, University Bordeaux-Sciences et Technologies, France and University West Macedonia Tei Kozani, Greece.

Prof. Ph.D. Eng. Dontu has published over 130 scientific papers in mechatronics field and laser processing technologies, being member of Romanian Mechatronics Society, International Mechatronics Society and International Laser Club.

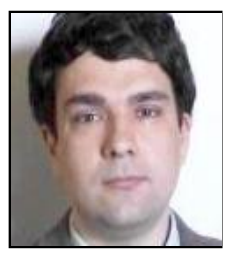

C.D. Comeaga was born in Bucharest, Romania, 1971. Constantin D. Comeaga received BSc. in Mechanical Engineering in 1994 and the PhD degree in technical acoustic and mechanical vibrations in 1999 from Politehnica University of Bucharest, Romania. The major fields of study are mechatronics, vibration and noise emission control, smart structures and robotics.

He works as a professor at University Politehnica of Bucharest, Romania, Faculty of Mechanical Engineeringa and Mechatronics, Department of Mechatronics. He is scientific director of Research and Development Center for Mechatronics.Prof. Ph.D. Eng. Comeaga's previous research activity deals with ultrasonic robotics, inch-worm motors, hysterezis and non-linearities compensation in piezoelectric actuators, semi-active control of damping using composite structures with piezoelectric patches. Current research deals with piezoelectric and electromagnetic actuated MEMS, smart sensors and electrodynanic actuators for vibration control. 


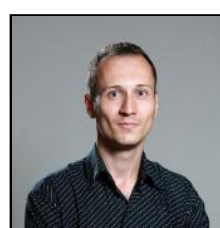

V.I. Gheorghe was born in Bucharest, Romania, 1977.

He received his BSc. in Mechanical Engineering and Mechatronics from Politehnica University of Bucharest, Romania, in 2003, his MSc in Advanced Mechatronics in 2004 and the $\mathrm{PhD}$ degree in Mechanical Engineering from Politehnica University of Bucharest, Romania, in 2012. He works as a Teaching Assistant at University POLITEHNICA of Bucharest, Romania, Faculty of Mechanical Engineering and Mechatronics, Department of Mechatronics and Precision Mechanics.

Univ. As. Ph.D. Eng. Gheorghe's research interests lie in the area of optomechatronics and biomimetic robots. Current research deals with electromagnetic actuated MEMS, and bioinspired mobile robotic structures. 